\title{
Die neue Grundlagenphilosophie von 1789 und die Versuche ihrer Verwirklichung in den Steuerordnungen der deutschen Staaten
}

\subsection{Die neuen liberalen Anforderungen und ihre theoretische Umsetzung}

\subsubsection{Die Ideale des Finanzliberalismus}

Für den klassischen Individualismus war der Staat ein Übel, und die „Besteuerung in jeder Form ist nichts weiter als eine Wahl von Übeln ${ }^{4437}$, denn fiskalische Eingriffe in die Volkswirtschaft fesselten die Entfaltung der Lebensenergien, hemmten den Fortschritt und minderten den Wohlstand. ${ }^{438}$

Ebenso wenig dürfe eine verantwortungsbewusste Regierung Schulden machen; denn aus Staatsschulden entstünden Steuern, was Elend und Unterdrückung zur Folge habe. ${ }^{439}$ Nicht nur Thomas Jefferson (1743-1826) hat unter dem Einfluss der französischen Aufklärung diesen Standpunkt vertreten, sondern auch der erste Präsident der Vereinigten Staaten von Amerika, George Washington (1732-1799).

437 Ricardo, David: Grundsätze der Volkswirtschaft und Besteuerung, 2. Aufl., Jena 1921, S. 163.

438 Mann, Fritz Karl: Steuerpolitische Ideale, S. $204 \mathrm{f}$.

439 Thomas Jefferson an Samuel Kercheval, Monticello, den 12.07.1816, zitiert nach: Mann, Fritz Karl: Steuerpolitische Ideale, S. 206 
In seiner Abschiedsbotschaft plädierte er für einen sparsamen Staat, denn wer Schulden tilgen wolle, müsse auch bereit sein, Steuern zu zahlen. ${ }^{440}$

Mäßigkeit der Besteuerung sei der Kern einer richtigen Finanzpolitik, so wiederholten es die Liberalen, aber auch die wissenschaftlichen Schriftsteller. ${ }^{441}$ Die Argumente, die Adam Smith für den wirtschaftlichen Individualismus geprägt hatte, wurden in Frankreich wesentlich von Jean-Baptiste Say (1767-1832) aufgegriffen und verbreitet.

In England wurde das individualistische Ideal der Beschränkung der Staatsfunktion maßgeblich durch die liberalen Schriftsteller David Ricardo (1772-1823) und Jeremias Bentham (1748-1832) vertreten. Ricardo sah in der allgemeinen Ein-

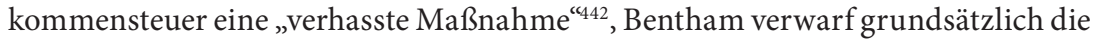
direkten Steuern und pries die Überlegenheit der indirekten Abgaben.

Die „Gewalt der finanzpolitischen Lage ${ }^{443}$ führte jedoch in England zur Income Tax durch Premierminister William Pitt (1759-1806) in den Jahren 1799 bis 1802, die durch Premierminister Henry Addington (1757-1844) 1803 erneut erhoben wurde. Nach Abschluss der Napoleonischen Kriege wurde diese Income Tax wieder abgeschafft. Sie war das Vorbild der im Jahr 1842 durch Premierminister Robert Peel (1788-1850) eingeführten Einkommensteuer, die helfen sollte, die chronischen Fehlbeträge des Staatshaushaltes zu beseitigen. ${ }^{444}$ Die Steuer überlebte wegen ihres großen Erfolges bei der Erhöhung der Staatseinnahmen, doch sie blieb weiterhin umstritten. Schatzkanzler William Ewart Gladstone (1809-1898) hielt vor dem englischen Parlament im Jahr 1862 seine berühmt gewordene pragmatische Rede, in der er seine Ansicht von der politischen Mission der Einkommensteuer wiedergab:

Wir stehen vor der alten Streitfrage um die direkte und die indirekte Steuer [...] Immer wenn ich an direkte und indirekte Steuern denke, kommen mir zwei anziehende Schwestern in den Sinn - die beide dieselben Eltern haben [...] nämlich die Notwendigkeit und die Findigkeit. ${ }^{445}$

440 Washington, George, Abschiedsrede am 17.09.1796, in: Senatsdokument Nr. 3, 102. Kongress, 1. Sitzung. Washington D. C. 1991.

441 Mann, Fritz Karl: Steuerpolitische Ideale, S. $212 \mathrm{ff.}$

442 Ebd., S. 228.

443 Ebd., S. 229.

444 Ebd., S.230f.

445 Dowell, Stephen: A History of Taxation and Taxes in England. From the Earliest Times to the Present Day, London 1888, ND, 3. Aufl., New York 1965, S. 365 und S. 369; Textstelle übersetzt von Eckart Schremmer: Über „gerechte Steuern“, S. 17. 
Schatzkanzler Sir Stafford Northcote setzte sich dagegen im Jahr 1874 für eine dauernde Beibehaltung der Income Tax ein, obwohl ein Budgetüberschuss existierte, der zu diesem Zeitpunkt den Verzicht erleichtert hätte. ${ }^{446}$ Zunächst vereinzelt und erst später verstärkt wurde die Einkommensbesteuerung durch die angelsächsischen Schriftsteller nicht nur aus Nutzenerwägungen, sondern auch als ethisches Postulat gerechtfertigt und anerkannt. ${ }^{447}$

\subsubsection{Die objektive Ertragsteuer}

Nach 1789 wurde eine Steuer gesucht, die dem neuen liberalen Denken entsprach. Diese Steuer sollte die bestehende Einkommens- und Vermögensverteilung - die als Bestandteil der natürlichen Ordnung und somit als gerecht angesehen wurde durch die Besteuerung nicht verändern und nach der steuerlichen Leistungsfähigkeit auf der Basis der Proportionalität verteilt werden. Die Merkmale dieser als gerecht angesehenen Steuer waren demnach: direkt, allgemein und gleichmäßig, proportional und neutral, d.h., es sollten keine außerfiskalischen Zwecke erfüllt werden. ${ }^{448}$

\subsubsection{Die Einheitstheorien}

Obwohl das neue System der objektiven Ertragsteuern in Form von Grund-, Gebäude- und Gewerbesteuer den liberalen Anforderungen des 19. Jahrhunderts gut zu entsprechen schien, hielt der Einfluss der physiokratischen Schule bis in das 19. Jahrhundert hinein auch in Deutschland an. Theodor Schmalz, der erste Rektor der im Jahr 1809 gegründeten Humboldt-Universität zu Berlin trat entschieden für eine einzige auf die Grundbesitzer nach dem Verhältnis des Reinertrages ihrer Grundstücke umgelegte Steuer als ideale Besteuerungsform ein. ${ }^{449}$

Der Gießener Professor Heinrich Wilhelm Crome griff 1817 den vom Grafen Julius von Soden 1808 und 1811 vorgetragenen Gedanken einer allgemeinen Produktensteuer auf. Er erklärte das Produkt, unter dem er „alles, was Geistes- und Körperkraft unter Mitwirkung der Natur hervorbringt"verstand, als den alleinigen

446 Haensel, Paul: Der Staatshaushalt und das Finanzsystem Großbritanniens, in: Gerloff, Wilhelm/ Meisel, Franz (Hrsg.): Handbuch der Finanzwissenschaft, Bd. 3, Tübingen 1929, S. 70-102, S. 76-79.

447 Mann, Fritz Karl: Steuerpolitische Ideale, S. 246.

448 Schremmer, Eckart: Über „gerechte Steuern“, S. 17.

449 Schmalz, Theodor/Thaer, Albrecht Daniel (Hrsg.): Encyclopädie der Cameralwissenschaften, Königsberg 1819, S. 772 f.; vgl. auch Krug, Leopold: Abriss der Staatsökonomie oder Staatswirthschaftslehre, Berlin 1808, S. 180 f., $\$ 194$. 
Gegenstand der Besteuerung. ${ }^{450}$ Das Maß der Steuerpflicht des Einzelnen wurde entsprechend durch den Wert der von ihm hervorgebrachten Produkte bestimmt. ${ }^{451}$

Mehrere Autoren ${ }^{452}$ sahen das „Vermögen“" als Grundlage und Maßstab einer einzigen direkten Steuer. Claus Kroncke wollte das „positive Vermögen“ des Einzelnen und damit alle drei von Smith übernommenen Produktionsfaktoren Boden, Arbeit und Kapital erfassen. Seinen Gedanken nahm der Erlanger Professor Johannes Paul Harl auf, der den Umfang der Steuerpflicht des Einzelnen allein nach der dem Wert des rechtlichen Staatsschutzes entsprechenden Größe des Vermögens des Bürgers bemaß. Mit dieser allgemeinen Vermögensteuer glaubte er ein ideell vollkommen gleichförmiges Steuersystem zu verwirklichen, ein Steuerideal, das die bisher erhobenen direkten und indirekten Abgaben ersetzen konnte. Der Gedanke einer einzigen direkten Vermögensteuer wurde in der ersten Hälfte des 19. Jahrhunderts noch von den Autoren Georg Gottfried Strelin, Dietrich Breitenstein, Karl von Rotteck und Karl Krehl getragen. ${ }^{453}$

Zusammenfassend kann man feststellen, dass die aufgeführten Einheitssteuertheorien nur dem Namen nach eine einzige Steuer darstellten; in Wirklichkeit handelte es sich bei ihnen um eine größere oder geringere Anzahl verschiedener Steuerformen. ${ }^{454}$

Im Gegensatz zu den Autoren der physiokratischen Schule stellten die Vertreter der allgemeinen Produkten- bzw. Vermögensteuer auf das Roheinkommen ab, was einen Rückschritt gegenüber der Erkenntnis der französischen und englischen Volkswirtschaftslehre bedeutete, denen zufolge die Steuern nur auf die reinen Einkünfte begründet werden dürfen, damit das nationale Wirtschaftsleben nicht gefährdet werde. ${ }^{455}$ Positiv hervorzuheben ist, dass all diese idealen Steuersysteme den Keim der Entwicklung eines theoretischen Steuersystems enthielten. Entgegen der in der Vergangenheit üblichen isolierten Betrachtung wurden die wechselseitige Bedingtheit der verschiedenen Steuerarten, ihre Funktionen und ihre Wirkungen unter einem einheitlichen Gesichtspunkt erörtert und zusammengefasst. Der Heidelberger Professor Friedrich Seeger hat das Wesen und die Bedeutung eines aus einer Anzahl direkter Steuern zusammengestellten Systems der Besteuerung

450 Crome, Heinrich Wilhelm: Das Steuerwesen aus rechtlichen Gesichtspunkten betrachtet, Hildesheim 1817, S. 33.

451 Wilke, Gustav: Die Entwicklung der Theorie, S. 21.

452 Ebd., S. 21-28.

$453 \mathrm{Zu}$ den Autoren und ihren Werken vgl. Wilke, Gustav: Die Entwicklung der Theorie, S. 21-28.

454 Ebd., S. 28.

455 Ebd., S. 21. 
zum ersten Mal erkannt und wies auf die Funktionen einer Ausgleichsteuer hin, die den ungleichen Belastungen entgegenwirken würde. ${ }^{456}$

\subsubsection{Die Entwicklung eines umfassenden Steuersystems}

Die Lehre von Adam Smith machte in Deutschland zuerst Georg Sartorius im Jahr 1796 in seinem „Handbuch der Staatswirthschaft“ bekannt. Sartorius teilte - wie verschiedene andere deutsche Autoren, wie Jakob Kraus, Georg von Buquoy und Stocker von Neuforn - die Lehre Smiths, von der er jedoch teilweise abging, als an ihn praktische Aufgaben herantraten. In seinem 1815 veröffentlichten Buch „Ueber die gleiche Besteuerung der verschiedenen Landesteile des Königreichs Hannover“ empfahl er im Interesse des gestiegenen königlichen Bedarfs außer der Grundsteuer und den Verbrauchsabgaben noch eine mäßige Gewerbe- sowie eine als Hilfssteuer gedachte Einkommensteuer. ${ }^{457}$

Auf der Grundlage der Smith'schen Lehre baute Johann Friedrich Eusebius Lotz ein System der direkten Besteuerung auf, mit dem er das gesamte Reineinkommen des Steuerpflichtigen im Wesentlichen erfassen und die Belastung gleichmäßig und allgemein durchführen wollte. ${ }^{458}$ Die Steuerlehre von Lotz bedeutete einen wesentlichen Fortschritt gegenüber der von Adam Smith entwickelten Kombination von Grund- und Konsumtionsabgaben, da er die zunehmende industrielle Entwicklung berücksichtigte und auch die aus den anderen Zweigen produktiver Tätigkeit erzielten Reineinkommen der direkten Besteuerung unterwerfen wollte. Doch gelang ihm die organische Eingliederung vor allem der Kapitalrentenbesteuerung ${ }^{459}$ in das Steuersystem nicht, sodass die Basis zu eng war, um eine größere Bedeutung in der Weiterentwicklung eines direkten Steuersystems einzunehmen. ${ }^{460}$

Erst der Hallenser Professor Ludwig Heinrich von Jakob - der dem theoretischen Steuersystem eine breitere Grundlage gab - führte diese Entwicklung zu einem

456 Ebd., S. 29.

457 Wilke, Gustav: Die Entwicklung der Theorie, S. 31.

458 Seeger, Friedrich: Versuch über das vorzüglichste Abgaben-System, Heidelberg 1811; vgl. Lotz, Johann Friedrich Eusebius: Handbuch der Staatswirthschaftslehre, Bd. 3, Erlangen 1822, S.304f.

459 Unter Kapitalrenten-Steuer oder Kapitalsteuer wurde eine Besteuerung der „Darlehensund anderer ähnlicher Zinsen und Renten“verstanden. Die Frage nach der Rechtmäßigkeit und Ausführbarkeit einer solchen Steuer ist von fast allen Finanzwissenschaftlern behandelt, aber von jedem anders beantwortet worden. Vgl. Vocke, Wilhelm: Ueber Kapitalrentenbesteuerung mit besonderer Rücksicht auf Bayern. Artikel. Ueber Kapitalrentenbesteuerung im Allgemeinen, in: Zeitschrift für die gesamte Staatswissenschaft 24, 1 (1868), S. 47-79, S. 47.

460 Wilke, Gustav: Die Entwicklung der Theorie, S. 35. 
vorläufigen Abschluss. In seiner 1821 erstmalig erschienenen „Staatsfinanzwissenschaft" ging er vorwiegend von volkswirtschaftlichen Gesichtspunkten aus und entwickelte das erste selbstständige geschlossene System einer Finanzwissenschaft. ${ }^{461}$ Ebenso wie Smith wollte er als Grundlage und Maßstab einer gerechten Steuerlastverteilung nur das reine Einkommen belasten, nicht jedoch das Stammvermögen oder werbende Vermögen, damit die Möglichkeit dauernder Erhebung der Abgaben gewahrt bleibe.

Von Jakob wollte alles Reineinkommen ohne Rücksicht auf seinen Ursprung besteuert wissen - im Gegensatz zu Smith, der im Wesentlichen nur die Grundrente besteuert wissen wollte. Die Smith'schen Produktionsfaktoren Boden, Kapital und Arbeit teilte er in eine Grundrente, Kapitalzinsrente und Industrierente. ${ }^{462}$

Die Industrierente teilte er - je nachdem, ob sie mit oder ohne Kapital erzielt wird - in persönlich sachliche (Unternehmergewinn) und persönliche (Arbeitslohn). ${ }^{463}$ Aus Gründen der Gerechtigkeit ${ }^{464}$ forderte Jakob eine gleichmäßige Erfassung sämtlicher Renten durch direkte Besteuerung - auch in Anbetracht der an Bedeutung zunehmenden Kapitalzinsen. ${ }^{465}$ Auch im Interesse einer einseitigen Belastung einzelner Produktionsfaktoren hielt er die gleichmäßige Erfassung sämtlicher Renten für erforderlich und gab für die Berechnung der Steuerbelastungen ausführliche und wohlbegründete Regeln vor. ${ }^{466}$

Im Gegensatz zu von Jacob forderte John Stuart Mill zur Verwirklichung des Smith'schen Steuergrundsatzes (Gleichmäßigkeit der Besteuerung) die steuerliche Freistellung des Sparanteils bei dem „terminable income“ (z. B. Unternehmergewinn oder Arbeitslohn), da der Empfänger von „permanent income“ Rücklagen für die Familie und für einkommenslose Zeiten bilden müsse. ${ }^{467}$ Er begründete dies damit, dass eine undifferenzierte Einkommensbesteuerung zu einer Doppelbelastung des Sparanteils führe, da die daraus erzielten Erträge wiederum der Besteuerung unterlägen, hingegen ausgegebenes Einkommen nicht mehr besteuert würde. ${ }^{468} \mathrm{Da}$ nicht nur das „terminable income“, sondern auch das „permanent income“ (z. B. Erträge aus Land- oder Wertpapierbesitz) von dieser Doppelbelastung betroffen waren, befürwortete Mill grundsätzlich eine Einkommensteuer, bei der die Ersparnisse

461 Ebd., S. 35.

462 Jakob, Ludwig Heinrich von: Die Staatsfinanzwissenschaft, Bd. 1, §587.

463 Ebd., $\$ \$ 663-667$.

464 Ebd., $\$ \$ 37,461$.

465 Ebd., $\$ \$ 604,609,645-653,679-680$.

466 Ebd., $\$ \$ 604-609$.

467 Mill, John Stuart: Principles of Political Economy, S. 815.

468 Ebd., S. 816. 
von der Steuer ausgenommen würden. ${ }^{469}$ Einschränkend fügte Mill jedoch hinzu, dass dies zu erhöhter Steuerhinterziehung führen könne, da diese Spareinlagen schwierig zu überprüfen seien. Aufgrund seiner Erkenntnis, dass Empfänger von "terminable income" normalerweise 25 Prozent von diesem Einkommen ansparen, schlug er vor, den Steuersatz für dieses Einkommen um ein Viertel tiefer anzusetzen als für das „permanent income“. 470

Von Jakob wollte ausschließlich den Reinertrag der Einkommensquellen erfassen, deshalb verwarf er konsequent die Personalsteuern wie Kopf- und Rangsteuern ${ }^{471}$ sowie die Vermögensteuern ${ }^{472}$ da diese weder mittelbar noch unmittelbar auf ein bestimmtes Reineinkommen Bezug nähmen. Er sah deshalb in der direkten Besteuerung die Grundlage und den Maßstab einer gerechten Verteilung der steuerlichen Abgaben, war sich jedoch sowohl der Unvollkommenheiten der Einkommensschätzungen als auch der Schwankungen des Einkommens bei den Steuerbürgern bewusst. Um diese unvermeidliche Ungerechtigkeit und Unzulänglichkeit der Veranlagung zu vermindern, wollte er einen Ausgleich durch eine teils direkte, teils indirekte Besteuerung durchführen. ${ }^{473}$ Die direkte Besteuerung des geringen Einkommens der unteren Volksklassen hielt er aus praktischen Erwägungen wegen der damit unverhältnismäßig hohen Kosten nicht für angemessen. ${ }^{474}$ Von Jakob forderte deshalb, die Konsumtionsteuern mit unterschiedlichen Prozentsätzen zu belegen und die Prozente bei verzichtbaren und wertvollen Gegenständen anzuheben. ${ }^{475}$ Die Konsumartikel der unteren Volksklassen sollten dabei mit geringeren Prozentsätzen belegt werden.

Das Steuersystem von von Jakob stellt das erste durchdachte, volkswirtschaftlich wohlbegründete und einheitlich geschlossene Besteuerungssystem dar, das der erste Versuch einer folgerichtigen Verbindung von direkten und indirekten Steuern unter einem einheitlichen Gesichtspunkt war. Dieses System stellte die konsequente Durchführung der individualistischen Staats- und Wirtschaftslehre der Steuerwissenschaft seiner Zeit dar und blieb auf Jahrzehnte hinaus die Grundlage der Theorie des Steuersystems. ${ }^{476}$

469 Ebd., S. 816.

470 Ebd., S. $816 \mathrm{f}$.

471 Jakob, Ludwig Heinrich von: Die Staatsfinanzwissenschaft, Bd. 1, §\$ 561-565.

472 Ebd., $\$ \$ 566-579$.

473 Ebd., $\$ \$ 683-697$.

474 Ebd., $\$ 698$.

475 Jakob, Ludwig Heinrich von: Die Staatsfinanzwissenschaft, Bd. 2, § 1112.

476 Wilke, Gustav: Die Entwicklung der Theorie, S. $38 \mathrm{f}$. 
Das von von Jakob vorgeschlagene System hielten Friedrich Karl von Fulda und der westfälische, spätere württembergische Minister Karl August Freiherr von Malchus für die beste Methode zur Erfassung des gesamten Reineinkommens der Steuerpflichtigen. Beide sahen als Quellen der reinen Einkünfte den Reinertrag des Bodens, des Kapitals und der Arbeit und mit der Kombination von Steuern auf den Ertrag dieser Produktionsfaktoren eine gerechte Belastung des gesamten Reineinkommens verwirklicht.

Von Fulda wollte als Ergänzung dieser Ertragsteuern die Konsumsteuern, die auf Güter des inneren (Akzise) sowie teilweise auf Güter des äußeren (Zölle) Verkehrs entfielen, mit eingliedern. Er sah darin - ebenso wie von Jakob - ein Mittel, um die Ungleichheiten, die durch die Unvollkommenheit der Einkommensteuern verblieben, auszugleichen. Dabei war bei den „Consumtionsteuern“"zu berücksichtigen, dass die ärmeren Klassen in geringeren Prozenten, und die wohlhabenderen Klassen mit höheren Tarifen belastet würden, um ein rechtes Maß der Verteilung vornehmen zu können. ${ }^{47}$

Von Malchus sah in der Nichterfassung der ohne Kapitalnutzung gezogenen reinen Arbeitsrente ein Gerechtigkeitsproblem, hielt jedoch die Konsumtionsbesteuerung für eine praktische Notwendigkeit, zumal auch die Erträgnisse der direkten Steuern einen hohen Finanzbedarf des Staates nicht ausgleichen konnten. Da nicht jede Art von Einkommen wegen der praktischen Begrenztheit der Schätzungsmethoden vollständig erfasst werden konnte, war nach seiner Ansicht auch aus Gerechtigkeitsgründen ein Ausgleich dieser Unvollkommenheiten durch die Besteuerung des Konsums geboten. ${ }^{478}$

Auch der Breslauer Professor Johann Schön sah ein aus Ertrag- und Verbrauchsteuern zusammengesetztes Steuersystem zur Erfassung des gesamten Reineinkommens des Steuerpflichtigen als gerecht und zweckmäßig an. Dabei fasste er die klassisch-liberalen Grundsätze der damaligen Zeit zusammen und betonte, dass die Steuern nur das reine Einkommen und nicht das Kapital erfassen dürften. Sie sollten weder für die Bürger lästig noch für den Staat kostspielig sein. Außerdem sollten die Steuergesetze so klar geregelt sein, dass die Steuerlast nur auf die im Gesetz geregelten Sachverhalte zutreffe und keinen anderen Steuerpflichtigen belastete. ${ }^{479}$

Den Abschluss dieser Entwicklung bildete Karl Heinrich Rau, dessen historischer Verdienst vorwiegend in der schärferen systematischen Zusammenfas-

477 Fulda, Friedrich Karl von: Handbuch der Finanzwissenschaft, Tübingen 1827, S. 274 ff.

478 Malchus, Carl August von: Handbuch der Finanzwissenschaft und Finanzverwaltung, 1. Theil: Finanzwissenschaft, Stuttgart/Tübingen 1830, S. 284-293.

479 Schoen, Johann: Die Staatswissenschaft, geschichts-philosophisch begründet (1831), 2. Aufl., Breslau 1840, S. 255. 
sung der wirtschaftstheoretischen Erkenntnisse seiner Zeit lag. ${ }^{480} 1855$ sah er das Gerechtigkeitspostulat mit der Anerkennung der gleichmäßigen Belastung aller Steuerpflichtigen als erfüllt an und betrachtete alle einzelnen Steuern als Teile eines Ganzen, die in ein richtiges Verhältnis zueinander gesetzt werden müssten. ${ }^{481}$

Da die praktische Durchführung einer einzigen allgemeinen Einkommensteuer an der Verschiedenartigkeit der Einkommenszweige scheitern würde, wollte Rau diese ebenso wie von Jakob, von Malchus und Schön als Hilfssteuer im Steuersystem gelten lassen. ${ }^{482}$ Um der Forderung nach Gerechtigkeit durch eine gleichmäßige Belastung aller Steuerpflichtigen Genüge zu tun, sollte insbesondere der notwendige Lebensbedarf von der steuerlichen Belastung freigestellt werden. ${ }^{483}$

\subsubsection{Die ersten theoretischen Ansätze einer subjektiven allgemeinen Einkommensteuer}

Es stellt sich die Frage, worin sich bei der objektiven Ertragsteuer die Gerechtigkeit zeigte; denn die Leistungsfähigkeit konnte nur anhand von äußerlich erkennbaren objektiven Merkmalen für den Ertrag des Besitzes eines Bürgers geschätzt werden. Die Steuer haftete auf dem Objekt, ohne die persönlichen Verhältnisse des steuerpflichtigen Eigentümers oder Nutznießers zu berücksichtigen. ${ }^{484}$ Die Autoren der herrschenden Lehre empfanden wohl die Mängel der objektiven Ertragsteuer, denn Sartorius ebenso wie von Jakob, von Malchus und Schön wollten eine allgemeine Einkommensteuer als Hilfssteuer im Steuersystem gelten lassen. Deutlicher als bei diesen Autoren trat bei den Vertretern einer allgemeinen Vermögensteuer das Bestreben in den Vordergrund, die persönlichen Verhältnisse der Steuerpflichtigen zu berücksichtigen. ${ }^{485}$

Die entscheidenden Anstöße für den Aufstieg der Einkommensteuer ergaben sich auf der Basis der liberalen Ideologie aus den großen Umwälzungen des 19. Jahrhunderts. ${ }^{486}$ In England wurde sie zuerst 1799 durch den englischen Premierminister William Pitt für die Finanzierung des Krieges gegen Frankreich und nach

480 Wilke, Gustav: Die Entwicklung der Theorie, S. 44.

481 Rau, Karl Heinrich: Lehrbuch der politischen Oekonomie, Bd. 3, Grundsätze der Finanzwissenschaft, Zweite Abtheilung, 3. Ausgabe, Leipzig/Heidelberg 1855, $\$ 401$.

482 Ebd., $\$ 401$.

483 Ebd., $\$ \$ 421 \mathrm{f}$.

484 Schremmer, Eckart: Über „gerechte Steuern“, S. $18 \mathrm{f}$.

485 Wilke, Gustav: Die Entwicklung der Theorie, S. 51.

486 Schremmer, Eckart: Über „gerechte Steuern“, S. 20 
englischem Vorbild auf Initiative des Feiherrn von und zum Stein nach 1808 und 1812 vorübergehend in Preußen eingeführt. ${ }^{487}$

Die theoretische Auseinandersetzung zugunsten einer allgemeinen Einkommensteuer begann mit einem kurzen Aufsatz über „Die Einkommen-Besteurung als das gerechteste Finanz-System“ in der im Jahr 1810 erschienenen Zeitschrift „Minerva“, in dem der Autor Leupert engagiert für eine progressive Einkommensteuer eintrat. ${ }^{488}$ Zwei Jahre später folgte der Erlanger Professor Alexander Lips mit seiner Schrift „Ueber die allein wahre und einzige Steuer, die Einkommen-Taxe und ihre Ausführbarkeit“, in der er eine Einkommensteuer mit sechs, das Einkommen nach seinen verschiedenen Quellen unterschiedlich belastenden Klassen empfahl. ${ }^{489}$ Heinrich Kessler setzte sich 1821 in seinem - „Finanzsystem nach den Verhältnissen Württembergs“ - ebenso wie Wilhelm Joseph Behr in seiner „Lehre von der Wirthschaft des Staats oder Pragmatische Theorie der Finanzgesetzgebung und Finanzverwaltung ${ }^{“ 490}$ für eine einzige direkte Einkommensteuer ein.

Weitere Unterstützung erfuhr die Einkommensteuer durch die angelsächsischen Schriftsteller, denn die allgemeine Einkommensteuer sollte nicht nur als wirtschaftliches, sondern auch als ethisches Postulat gerechtfertigt werden. In einer Ausgabe der „Edinburgh Review“ aus dem Jahr 1833 war der Satz zu lesen: „Keine Steuer ist eine gerechte Steuer, sofern sie die Individuen nicht in derselben Lage läßt, in der sie sie vorfindet. ${ }^{{ }^{491}}$ Dieses Postulat verbreitete sich schnell unter der treffenden Bezeichnung „leave-them-as-you-find-them-rule of taxation “492 und wurde als klare Absage an die Progression verstanden. John Stuart Mill forderte aus Gründen der Steuergleichheit die steuerliche Freistellung des Existenzminimums. Mit der Freistellung des Existenzminimums ergäbe sich bei einer proportionalen Tarifstruktur ein indirekt progressiver Effekt, der dem Gleichheitsgebot genügend Rechnung trage. Aus diesem Grund sei eine allgemeine Steuerprogression nicht erforderlich. Da außerdem eine allgemeine Steuerprogression nicht wissenschaftlich

487 Die 1808 für die Provinz Ostpreußen und Litauen eingeführte Einkommensteuer wurde 1812 auf den Gesamtstaat ausgedehnt, jedoch 1820 durch eine Klassensteuer ersetzt, vgl. Schwennicke, Andreas: „Ohne Steuer kein Staat“, S. 337.

488 Leupert (Rechnungs-Kalkulator): Die Einkommen-Besteurung als das gerechteste Finanzsystem (Blos im Entwurf), in: Minerva. Ein Journal historischen und politischen Inhalts, Johann Wilhelm von Archenholz (Hrsg.) 3. Bd., Juli, August, September, Berlin 1810, S. 38-52.

489 Lips, Alexander: Ueber die allein wahre und einzige Steuer.

490 Behr, Wilhelm Joseph: Die Lehre von der Wirthschaft des Staats oder Pragmatische Theorie der Finanzgesetzgebung und Finanzverwaltung, Leipzig 1822.

491 Zitiert nach Mann, Fritz Karl: Steuerpolitische Ideale, S. 246.

492 Ebd., S. 247: Die Edinburgher Regel, ein Postulat der Besteuerungsneutralität. 
begründet werden könne und auch derjenige, der mehr leiste noch bestraft werden würde, lehnte Mill eine progressive Tarifstruktur ab. ${ }^{493}$ Unter diesem Gesichtspunkt erschien die proportionale Einkommensteuer zur damaligen Zeit alternativlos. ${ }^{494}$

\subsection{Der Zustand in den deutschen Staaten bis zur Verfassung des Deutschen Reiches vom 28. März 1849}

\subsubsection{Die Napoleonischen Kriege und ihre finanziellen Folgen}

Zwischen 1792 und 1815 gerieten die öffentlichen Finanzen aus dem Gleichgewicht, was vor allem an den Revolutions- und Napoleonischen Kriegen lag. Die deutschen Staaten mussten ihre Militärausgaben steigern und mehr als zwei Jahrzehnte auf hohem Niveau halten. Zum wichtigsten Mittel, diese Finanzschwierigkeiten $\mathrm{zu}$ bewältigen, entwickelte sich die Verschuldung, es kam zu einer regelrechten Schuldenexplosion. ${ }^{495}$ Die meisten deutschen Staaten gingen in verschleierter Form einmal oder mehrfach bankrott. Sie bedienten ihre Schulden teilweise verspätet oder gar nicht. Fast alle Staaten erkannten, dass sie ihr Finanzwesen von Grund auf umgestalten mussten; diese Reformen des frühen 19. Jahrhunderts waren ein Kind der Krise. ${ }^{496}$ In einer Zeit der finanziellen Krise galt es als vordringlichstes Ziel, die staatlichen Einnahmen zu erhöhen. Überall im Staat sollten dieselben Steuern erhoben und nach der Leistungsfähigkeit bemessen werden. ${ }^{497}$

Die rechtlichen Schranken fielen 1806 mit dem Ende des Heiligen Römischen Reiches Deutscher Nation. Unter dem Druck von Napoleon Bonaparte schloss sich eine Reihe deutscher Staaten zu einem Staatenbund zusammen, der sich als Rheinbund bezeichnete. Im Gefolge der Rheinbundgründung legte Kaiser Franz II. am 6. August 1806 die deutsche Kaiserkrone ab. Das Heilige Römische Reich Deutscher Nation hatte damit aufgehört zu bestehen. ${ }^{498}$

Nun waren auf dem Territorium des ehemaligen Heiligen Römischen Reiches Deutscher Nation unterschiedliche Staatengruppierungen entstanden. Die erste

493 Mill, John Stuart: Principles of Political Economy, S. 810 f.

494 Mann, Fritz Karl: Steuerpolitische Ideale, S. 247.

495 Ullmann, Hans-Peter: Der deutsche Steuerstaat, S. 24.

496 Ebd., S. 25.

497 Ebd., S. $27 \mathrm{f}$.

498 Schlosser, Friedrich Christoph: F. C. Schlossers Weltgeschichte für das deutsche Volk. Bd. 18, Frankfurt am Main 1856, S. 113 f. 
Gruppierung bestand aus dem Kaiserreich Österreich mit den nationalen Gebieten, die zweite bestand aus dem Rheinbund und die dritte aus einzelnen Staaten bzw. staatlichen Gebilden.

\subsubsection{Die Bemühungen preußischer Politiker auf wirtschafts- und steuerpolitischem Gebiet, um die Souveränität des Königreiches wiederzuerlangen}

Das Königreich Preußen war als größter Bundesstaat unter diesen Gegebenheiten nicht nur isoliert, sondern auch das politische und militärische Ziel des französischen Kaisers. Einer militärischen Auseinandersetzung mit Frankreich war Preußen aus vielerlei Gründen nicht gewachsen, sodass es zwischen Oktober 1806 und Juni 1807 besiegt werden konnte. Im Juni 1807 kam es zu Friedensverhandlungen in Tilsit, in denen Napoleon Bedingungen stellte, die für das Königreich verheerend waren. Preußen verlor nicht nur einen großen Teil seines Territoriums, sondern wurde neben politischen und militärischen Demütigungen zu außerordentlichen Kontributionen und Leistungen für die französischen Streitkräfte verpflichtet. ${ }^{499}$ Napoleon machte zur Bedingung, dass er seine Truppen erst aus Preußen abziehen werde, wenn die Kontributionen in Höhe von 12 Millionen Francs beglichen seien. ${ }^{500}$ Es war deshalb ein hochpolitisches Anliegen der preußischen Staatsmänner, dafür zu sorgen, dass diese Kontributionen möglichst bald und vollständig aufgebracht wurden. Das war jedoch nicht ohne Weiteres möglich, denn nach dem Tilsiter Vertrag hatte das Königreich Preußen 54 Prozent seiner Einwohner und mehr als die Hälfte seines Territoriums verloren. ${ }^{501}$

Es war keine leichte Aufgabe, dem verarmten Land wesentlich höhere Steuerlasten als vor 1806 aufzuerlegen, die zahllosen verschiedenen Steuersysteme in ein einheitliches zu verwandeln und dabei das gewachsene Finanzsystem von Grund auf zu reformieren. ${ }^{502}$

499 Schmoller, Gustav: Die Epochen der preußischen Finanzpolitik, Separatabdruck aus: Jahrbuch Gesetzgebung, Verwaltung und Volkswirtschaft 1 (1877), S.3-114, S. 80.

500 Kumpf, Johann Heinrich: 5000 Jahre Steuern und Zölle. Die finanzgeschichtliche Schausammlung der Bundesfinanzakademie in Brühl, Brühl 1996, S. 75.

501 Kleine Enzyklopädie Deutsche Geschichte, Leipzig 1965, S.216; Sahm, Reiner: 5000 Jahre Steuern, S. 224.

502 Schmoller, Gustav: Die Epochen der preußischen Finanzpolitik, S. 82. 
Für die preußische Staatsführung gab es nur zwei Möglichkeiten: Staatsbankrott oder die grundlegende Reform der Gesellschaft. ${ }^{503}$ Heinrich Friedrich Karl Reichsfreiherr von und zum Stein (Preußischer Handelsminister von Januar 1804 bis Januar 1807) beschloss, die Domänen zu veräußern, und führte in Ostpreußen und Litauen, dann auch in Westpreußen eine progressive Einkommensteuer zur Abzahlung der französischen Kontributionen ein. Dies war das erste deutsche Einkommensteuergesetz überhaupt. ${ }^{504}$

Nach diesem Reglement vom 23. Februar 1808 sollte

von sämmtlichen Eigenthümern und Einwohnern der Städte und des platten Landes in Ostpreußen und Lithauen, vom Militair- und Zivil-Stande, ohne alle Ausnahme als die, welche sich aus dem etwaigen gänzlichen Unvermögen von selbst ergiebt, eine Einkommensteuer so lange erhoben werden, bis das Kriegsschuldenwesen gänzlich getilgt seyn wird. ${ }^{505}$

Stein erklärte die Einkommensteuer für die billigste Abgabe, „da sie alle Staatsbürger und alle Quellen des Nationalwohlstandes gleichmäßig treffe. ${ }^{\text {“506 }}$ Auf die Opposition dürfe man keine Rücksicht nehmen. Es herrsche da - er meinte damit vor allem den kurmärkischen Adel - ein tief verwurzelter Egoismus, verbunden mit halber Bildung und gemütsloser Rohheit. ${ }^{507}$

Im Edikt über die Finanzen des Staats vom 27. Oktober 1810 hatte König Friedrich Wilhelm III. von Preußen (1770-1840) völlige Gewerbefreiheit gegen Entrichtung einer mäßigen „Patentsteuer“ bei gleichzeitiger Einstellung der bisherigen Gewerbesteuern angekündigt. Am Tag darauf wurde dann erstmals die allgemeine Gewerbesteuer in Preußen eingeführt, die 1820 neu gestaltet wurde..$^{508}$

Am 30. Oktober 1810 versuchte Karl August von Hardenberg, eine Luxus- und Verbrauchsteuer einzuführen, um auf diese Weise mit der Praxis des Steuerprivilegs zu brechen. Sein Vorhaben, auch eine allgemeine Grundsteuer zu erheben, scheiterte am Unwillen des Adels. ${ }^{509}$

503 Ziegler, Dieter: Das Zeitalter der Industrialisierung (1815-1914), in: North, Michael (Hrsg.): Deutsche Wirtschaftsgeschichte. Ein Jahrtausend im Überblick, 2. Aufl., München 2005, S. 197-286, S. 198.

504 Schmoller, Gustav: Die Epochen der preußischen Finanzpolitik, S. 84.

505 Zitiert in: Kumpf, Johann Heinrich: 5000 Jahre Steuern und Zölle, S. 75.

506 Schmoller, Gustav: Die Epochen der preußischen Finanzpolitik, S. 84.

507 Ebd., S. 84.

508 Kumpf, Johann Heinrich: 5000 Jahre Steuern und Zölle, S. 77.

509 Sahm, Reiner: 5000 Jahre Steuern, S. 226. 
Die geringen Erfolge bei dem Versuch, das Finanzsystem zu reformieren, führten in der Folgezeit zu einem Anwachsen der Staatsschulden. Diese betrugen Ende 1812 insgesamt 131,7 Millionen Thaler und stiegen bis zum Jahr 1820 auf 180 Millionen Thaler an.${ }^{510}$ Die Rückzahlung der Staatsschuld wurde als Ehrensache dargestellt, was in Anbetracht der Tatsache, dass die adligen Großgrundbesitzer, die die größten Gläubiger des Landes waren und maßgebenden Einfluss auf die preußische Politik hatten, nicht verwunderlich war. ${ }^{511}$

Die Steuerreformen zwischen 1818 und 1822 erwiesen sich fiskalisch als sehr ergiebig, jedoch wies das preußische Steuersystem massive Verstöße gegen den Gleichheitsgrundsatz auf. Die in 1820 eingeführte Mahl- und Schlachtsteuer ${ }^{512}$ bot den Kritikern des preußischen Steuersystems die größte Angriffsfläche. ${ }^{513}$

Wegen ihrer unsozialen Wirkung war die Mahl- und Schlachtsteuer starker Kritik ausgesetzt. Durch die Mahlsteuer wurde Weizenmehl wesentlich stärker als andere Getreideprodukte belastet, was dazu führte, dass ärmere Stadtbewohner auf Roggenmehl oder auf die steuerlich unbelastete Kartoffel als Massengrundnahrungsmittel ausweichen mussten. Auch bei der Schlachtsteuer konnten Wohlhabende von besteuertem Großviehfleisch auf Geflügel, Wildbret oder Fisch ausweichen, der ärmeren Bevölkerung blieb hingegen nur der Konsumverzicht, um der Schlachtsteuer zu entgehen. ${ }^{514}$ Beide Steuern führten dazu, dass die Steuerlast pro Kopf in den mahl- und schlachtsteuerpflichtigen Städten etwa dreimal höher war als die Klassensteuer ${ }^{515}$ - eine Mischung aus Kopfsteuer und übrig gebliebe-

510 Schmoller, Gustav: Die Epochen der preußischen Finanzpolitik, S. 81.

511 Spoerer, Mark: Steuerlast, Steuerinzidenz und Steuerwettbewerb. Verteilungswirkungen der Besteuerung in Preußen und Württemberg (1815-1913) (Jahrbuch für Wirtschaftsgeschichte, Beiheft 6), Berlin 2004, S. 131.

512 Die Mahlsteuer wurde von dem zur Mühle gebrachten Getreide und den in das Steuergebiet eingeführten Fertigwaren erhoben; die Schlachtsteuer von den zum Schlachten angelieferten Tieren bzw. den eingeführten Fleisch- und Fettwaren.

513 Spoerer, Mark: Steuerlast, S. $131 \mathrm{ff}$.

514 Ebd., S. 132.

515 „Die Klassensteuer war von Anfang an eine antiquierte Steuer, da sie die Leistungsfähigkeit der Steuerpflichtigen nach ihrer Standeszugehörigkeit einschätzte." Spoerer, Mark: Steuerlast, S. 47. Die Klassensteuer teilte die Steuerpflichtigen nach äußeren Merkmalen, von denen sich auf die individuelle Leistungsfähigkeit schließen ließ, in vier Klassen mit gestaffelten Sätzen ein; denn sie sollte „Zwischen einer ohne genaues Eindringen in die Vermögensverhältnisse der Pflichtigen nicht ausführbaren und deshalb immer gehässigen Einkommensteuer und einer die Gesamt-Masse aller Einwohner ohne allen Unterschied gleich treffenden Kopfsteuer die Mitte halten." Vgl. Kellenbenz, Hermann: Deutsche Wirtschaftsgeschichte, Bd. 2: Vom Ausgang des 18. Jahrhunderts bis zum 
ner Einkommensteuer -, die 1820 für das „platte Land“ und die kleineren Städte eingeführt worden war. ${ }^{516}$

Ein weiterer gravierender Verstoß gegen den Gleichheitssatz ergab sich daraus, dass die Behörden um die mahl- und schlachtsteuerpflichtige Stadt eine Steuerlinie - anhand vorhandener Befestigungen, eines Flusses etc. - festlegten. Viele Städte waren jedoch über die mittelalterlichen Befestigungsmauern hinausgewachsen, sodass eine weitere, äußere Steuerlinie gezogen wurde, was zur Auswirkung hatte, dass die zwischen den Linien wohnenden Menschen einer doppelten Besteuerung unterworfen wurden. Diese mussten nicht nur die Klassensteuer zahlen, sondern auch über die Brot- und Fleischpreise die darin enthaltende Mahl- und Schlachtsteuer entrichten. Hinzu kam, dass die meisten Städte neben der staatlichen Mahl- und Schlachtsteuer einen Kommunalzuschlag erhoben, dessen Durchschnitt sich im Laufe der Jahre der maximal zulässigen Grenze von 50 Prozent näherte. ${ }^{517}$

Das Prinzip der gleichen Steuerlast bei gleichem Steuertatbestand hatte Preußen bewusst der fiskalischen Ergiebigkeit geopfert. ${ }^{518}$

\subsubsection{Die Bestrebungen in den deutschen Staaten um eine Modernisierung des Steuerwesens}

In den Rheinbundstaaten war man dem Vorbild Frankreichs gefolgt und hatte einen Modernisierungsschub erfahren. Das von Napoleon 1804 verfasste französische Zivilgesetzbuch, der Code civil oder Code Napoléon, kam auch in diesen Staaten zum Tragen und garantierte das Recht auf Privateigentum und andere bürgerliche und wirtschaftliche Freiheiten. ${ }^{519}$ In ganz Frankreich begann Napoleon 1807 mit der umfassenden Grundsteuerkatastrierung, die die Durchführung und Sicherung der Grundsteuer in die Wege leitete. Ein Jahr später kam die Personal- und Mobiliarsteuer (Wohnungssteuer) hinzu, die durch eine Gewerbe- (Patent-) und eine Gebäudesteuer in Form der Tür- und Fenstersteuer ergänzt wurde. Damit war

Ende des Zweiten Weltkriegs, München 1981, S. 157; Kumpf, Johann Heinrich: 5000 Jahre Steuern und Zölle, S. 77.

516 Spoerer, Mark: Steuerlast, S. 16 und S. 132 f.

517 Ebd., S. 135.

518 Wie tiefgreifend die preußischen Steuerreformen von 1818-1822 nicht nur eine Steuerlastverteilung von oben nach unten bewirkten, sondern auch zu einer Umverteilung von Ost nach West führten, zeigt im Einzelnen Spoerer, Mark: Steuerlast, S. 47-55 und S. 133.

519 Sahm, Reiner: 5000 Jahre Steuern, S. 226. 
das Ertragsteuersystem mit Grund-, Gewerbe- und Gebäudesteuern geschaffen, das in Frankreich über 100 Jahre lang in den Grundzügen bestehen blieb. Dieses System hat die Steuergesetzgebung in den westlichen und südlichen deutschen Staaten entscheidend beeinflusst. ${ }^{520}$ Inhaltlich ging es in erster Linie darum, die Steuergleichheit durchzusetzen. ${ }^{521}$

Im Gefolge des Wiener Kongresses kam es in den deutschen Staaten zu einigen grundlegenden territorialen Veränderungen sowie zum Entstehen des Deutschen Bundes. Da die wesentlichen Souveränitätsrechte den Mitgliedern des Deutschen Bundes überlassen waren, blieben dem Bund selbst nur geringfügige eigene finanzwirtschaftliche Funktionen. Der Deutsche Bund umfasste 35 Monarchien bzw. Fürstentümer und vier Freie Städte mit eigenem individuellem Finanzsystem, das Steuerbewilligungsrecht übernahmen die Parlamente. ${ }^{522}$

Es hatten sich in den deutschen Staaten zwei Grundformen der Steuersysteme gebildet. Die eine Variante - die sich in den süddeutschen Staaten im frühen 19. Jahrhundert entwickelt hatte - setzte auf eine Objektbesteuerung in Gestalt eines dreigliedrigen Ertragsteuersystems mit Grund-, Gebäude- und Gewerbesteuer. Diese Besteuerung stellte nicht auf den real erzielten, sondern auf einen durchschnittlichen, anhand äußerer Merkmale ermittelten Ertrag ab. Die Schwächen dieser Ertragsteuern lagen darin, dass sie mit der wachsenden Leistungsfähigkeit der Besteuerten nicht Schritt hielten und sich vom wirtschaftlichen Wachstum und zunehmenden Wohlstand abkoppelten. ${ }^{523}$ Die zweite Variante - die die Staaten in der Mitte und im Norden gewählt hatten - setzte weniger auf Ertrag-, sondern mehr auf Personalsteuern, ohne aber den Schritt zu einer modernen Einkommensteuer getan zu haben. ${ }^{524}$

Am 22. März 1833 schlossen sich der Preußisch-Hessische Zollverein, der Mitteldeutsche Handelsverein und der Süddeutsche Zollverein zum Deutschen Zollverein zusammen, was zur Folge hatte, dass unter dem Einfluss des Nationalökonomen Friedrich List die Binnenzölle bis zur Reichsgründung 1871 schrittweise zurückgedrängt wurden. Mit dem Deutschen Zollverein wurde ein einheitliches

520 Schmölders, Günter: Geschichte der Besteuerung, S. 621.

521 Kumpf, Johann Heinrich: 5000 Jahre Steuern und Zölle, S. 75.

522 Kellenbenz, Hermann: Deutsche Wirtschaftsgeschichte, S. 156; Sahm, Reiner: 5000 Jahre Steuern, S. $226 \mathrm{f}$.

523 Ullmann, Hans-Peter: Der deutsche Steuerstaat, S. 43 f.

524 Ebd., S. 44. 
deutsches Wirtschaftsgebiet geschaffen, was als Vorstufe zur politischen Einigung angesehen wurde. ${ }^{525}$

Es waren nicht nur die Privilegierten, die sich gegen die steuerpolitischen Vorhaben zur Wehr setzten. Auch innerhalb der bäuerlichen Bevölkerung und der städtischen Einwohnerschaft kam es immer wieder zur Auflehnung gegen neue steuerliche Belastungen. Branntwein-, Bier-, Mahl- und Schlachtsteuern breiteten sich aus, was zu offenem Widerstand der bäuerlichen Bevölkerung führte, die selbst vor tätlichen Angriffen auf Steuereinnehmer nicht zurückschreckte. In Schlesien entzündete sich 1811 der Volkszorn, bewaffnete Bauern zogen durch das Land, deren Widerstand gegen die Steuern erst nach Einsatz des Militärs unter Kontrolle gebracht werden konnte. ${ }^{526} 1830$ musste das Militär in Kurhessen eingesetzt werden, um gegen den offenen Aufruhr vorzugehen. Fast alle Schichten der Bevölkerung der Provinz Hanau beteiligten sich an dem „Mautensturm“, dem fast alle Zolleinrichtungen zum Opfer fielen. ${ }^{527}$

Die Revolution von 1848/49 begann und endete ebenfalls mit Steuerprotesten. So richteten sich die Agrarunruhen, die im März 1848 ausbrachen, auch gegen die staatlichen Abgaben, die vielfach als zu hoch und ungerecht verteilt empfunden wurden. ${ }^{528}$ Nach den Agrarunruhen im März riefen die Rustikalvereine dazu auf, die Steuerprivilegien der Landeigentümer abzuschaffen. Als Reaktion auf demütigende Entscheidungen des Königs beschloss die preußische Nationalversammlung am 15. November 1848, dass die preußische Regierung nicht mehr berechtigt sei, Steuern zu erheben oder über sie zu verfügen. Dies gab den Anstoß zu einer breiten Steuerverweigerungskampagne in den preußischen Provinzen, die im Wesentlichen von den Bonner Demokraten Carl Schurz (1829-1906) und Gottfried Kinkel (1815-1882) unterstützt wurde. ${ }^{529}$

Als eine der Ursachen der Steuerproteste wurde von dem ehemaligen preußischen Finanzminister David Hansemann im Jahr 1850 rückblickend festgestellt: „Daß der Grundsatz der gleichmäßigen Besteuerung [...] nicht längst zur Ausführung gekommen ist, betrachte ich als eine der Ursachen der Revolution. ${ }^{“ 530}$ Schon die Verfassungsentwürfe aus der Zeit vor dem Zusammentreten der Nationalversamm-

525 Lütge, Friedrich: Deutsche Sozial- und Wirtschaftsgeschichte, S. 467; Kumpf, Johann Heinrich: 5000 Jahre Steuern und Zölle, S. 89.

526 Ullmann, Hans-Peter: Der deutsche Steuerstaat, S. 49.

527 Ebd., S. 50.

528 Kumpf, Johann Heinrich: 5000 Jahre Steuern und Zölle, S. 78.

529 Ebd., S. 79; Sahm, Reiner: 5000 Jahre Steuern, S. 131.

530 Hansemann, David Justus Ludwig: Das Preußische und Deutsche Verfassungswerk. Mit Rücksicht auf mein politisches Wirken, 2. Aufl., Berlin 1850, S. 103. 
lung enthielten grundrechtliche Bestimmungen, die auch die Forderung nach einem Gleichheitssatz im Steuerrecht einschlossen. ${ }^{531}$ Das von der Nationalversammlung vorgefundene Steuerrecht in den einzelnen deutschen Staaten wies eine Fülle von Ungleichheiten und Begünstigungen auf, die sachlicher und persönlicher Art waren. Da die überkommenen Ungleichheiten von vielen Reformern als untragbar angesehen wurden, wurde bereits von der Vorkommission des Verfassungsausschusses die Formulierung von der „Gleichheit der Besteuerung für Personen und Sachen“ beschlossen. ${ }^{532}$

Die Nationalversammlung verabschiedete nach vielen kontroversen Debatten die Verfassung des deutschen Reiches vom 28. März 1849 (Paulskirchenverfassung). ${ }^{533}$ Danach enthielt der $\$ 173$ als lex specialis zu $\$ 137$ Abs. 3 („Die Deutschen sind vor dem Gesetze gleich.“) eine besondere Ausprägung des allgemeinen Gleichheitssatzes: „Die Besteuerung soll so geordnet werden, daß die Bevorzugung einzelner Stände und Güter im Staat und Gemeinde aufhört." Die Vorschrift des $\$ 173$ wurde einhellig als Gebot der Besteuerungsgleichheit und als Verpflichtung zur Rechtsetzungsund Rechtsanwendungsgleichheit im Steuerrecht aufgefasst, enthielt jedoch keine Vorgaben für die Ausgestaltung des künftigen Steuersystems. ${ }^{534}$

Mit der Verabschiedung der Paulskirchenverfassung wählten die Parlamentarier den preußischen König zum Kaiser der Deutschen. Der preußische König Friedrich Wilhelm IV. lehnte jedoch die Kaiserkrone und die Anerkennung der Reichsverfassung ab, was zu bewaffneten Auseinandersetzungen führte. Die Aufstände wurden niedergeschlagen, die Revolution in Deutschland fand mit der Kapitulation von Rastatt vor dem preußischen Korps am 23. Juli 1849 ihr Ende. ${ }^{535}$

Die Revolution von 1848/49 hatte jedoch den Effekt, dass sich das bürgerliche mit dem adligen Lager gegen die unteren Schichten vereinte, was den Weg für die erste Finanzreform Preußens seit drei Jahrzehnten ebnete. Mit dem am 1. Mai 1851 verabschiedeten Gesetz blieb zwar die Mahl- und Schlachtsteuer erhalten, doch die Klassensteuer wurde auf jährliche Einkommen bis zu 1.000 Talern beschränkt. Darüber hinausgehende Einkommen unterlagen einer neu geschaffenen klassifizierten Einkommensteuer, für die ein Pauschalabzug gewährt wurde, wenn die Steuerpflichtigen in einer mahl- und schlachtsteuerpflichtigen Stadt wohnten.

531 Kempny, Simon: Die Staatsfinanzierung nach der Paulskirchenverfassung. Eine Untersuchung des Finanz- und Steuerverfassungsrechts der Verfassung des deutschen Reiches vom 28. März 1849, Tübingen 2011, S. 164.

532 Ebd., S. $165 \mathrm{f}$.

533 RGBl. 1849, 16tes Stück, S. 101-148.

534 Kempny, Simon: Die Staatsfinanzierung, S. 184-189.

535 Sahm, Reiner: 5000 Jahre Steuern, S. 232. 
Beide Steuern beruhten auf einer Einschätzung nach äußeren Merkmalen, nach denen die Schätzungskommissionen die steuerbaren Einkommen festlegten. Die neu geschaffene klassifizierte Einkommensteuer brachte große Mehreinnahmen, ohne das Aufkommen aus der Klassensteuer nennenswert zu beeinträchtigen. ${ }^{536}$

1861 wurde die Grundsteuer in drei Gesetzen reformiert, was dazu führte, dass über 92 Prozent der Mehrbelastung von den östlichen Provinzen aufgebracht wurden und sich der Anteil der Grund- und Gebäudesteuer am gesamten Aufkommen der direkten Staatssteuern von insgesamt 38,4 Prozent auf 43,4 Prozent erhöhte. Die bedeutsamste Regelung des Grundsteuergesetzes war die Aufhebung der meisten Freistellungen, die aufgrund eines speziellen Titels grundsätzlich steuerpflichtige Personen eximierte. Als klassische Ertragsteuer des 19. Jahrhunderts orientierte sich diese Steuer nicht am tatsächlichen Bodenertrag, sondern an einem fiktiven, aus langjährigen Mittelwerten gebildeten Durchschnittssollertrag. ${ }^{537}$

Für Württemberg ergab sich für die regionale und sektorale Steuerlastverteilung ein ähnliches Bild wie für Preußen. Nach der Vereinheitlichung des Steuersystems 1821/23 führten die Reformen der direkten Steuern zu einer Steuerlastverlagerung in die zunehmend durch Gewerbe- und Dienstleistungen geprägten und überdurchschnittlich wachsenden Regionen - mit der Folge, dass die Steuerlast pro Kopf gesteigert wurde. ${ }^{538}$

\subsection{Zusammenfassung und Fazit}

\section{Zur Verwirklichung des Allgemeinheitsgrundsatzes der Besteuerung}

In den vorausgegangenen Jahrhunderten hatten die herrschenden Stände regelmäßig von verschiedenen steuerlichen Privilegien profitiert, was auch historisch bedingt war, denn Adel und Klerus hatten ohne Ausgleich Dienstleistungen militärischer, administrativer oder sozialer Art übernommen. Die weitgehenden Steuerprivilegien des Adels waren im 19. Jahrhundert vollkommen unbegründet, blieben jedoch vielfach wirksam und stellten damit eine grobe Verletzung des Allgemeinheitsgrundsatzes der Besteuerung dar. Bemerkenswert ist, dass dieser Grundsatz seinerzeit schon verfassungsrechtlich weitgehend anerkannt, als Programmsatz ausformuliert und somit ausdrücklich festgeschrieben worden war. $\mathrm{Zu}$ nennen sind vor allem die Königreiche Bayern und Sachsen und das Großherzogtum Baden, weiterhin aber

536 Spoerer, Mark: Steuerlast, S. 56 f.

537 Ebd., S. 60-67.

538 Ebd., S. 101. 
auch die Verfassungen des Königreichs Württemberg, der Groß- bzw. Herzogtümer Hessen, Sachsen-Meiningen und Sachsen-Altenburg. Dies hatte jedoch nicht zur Folge, dass das bestehende Privilegienwesen in den deutschen Staaten vollständig beseitigt wurde. So konnte zur damaligen Zeit von einer Verwirklichung des Allgemeinheitsgrundsatzes noch keine Rede sein. ${ }^{539}$

Obwohl der Grundsatz der Allgemeinheit der Besteuerung als Fundamentalprinzip eine Grundvoraussetzung einer gerechten und sozialen Finanzpolitik ist und seit der Französischen Revolution unübersehbar gefordert wurde, weigerten sich die herrschenden Stände im 19. und selbst zu Beginn des 20. Jahrhunderts, auf ihre Privilegien zu verzichten. Die Reichskirche hatte mit dem Reichsdeputationshauptschluss vom 25. Februar 1803 aufgehört zu existieren. Der größte Teil des kirchlichen Vermögens war säkularisiert worden.

\section{Zum Grundsatz der Gleichmäßigkeit der Besteuerung}

Auf der Grundlage der liberalen Denkweise von 1789 wurde eine Steuer gesucht, die den neuen liberalen Anforderungen entsprach. Diese Steuer sollte die bestehende Einkommens- und Vermögensverteilung - die als Bestandteil der natürlichen Ordnung und somit als gerecht angesehen wurde - nicht verändern und nach der steuerlichen Leistungsfähigkeit auf der Basis der Proportionalität verteilt werden. Die Merkmale dieser neuen gerechten Steuer waren demnach: direkt, allgemein und gleichmäßig, proportional und neutral, d.h., es sollten keine außerfiskalischen Zwecke erfüllt werden.

In der Literatur entstanden Einheitstheorien, die den Reinertrag des Bodens bzw. das „Vermögen“ als Grundlage und Maßstab einer einzigen Steuer ansahen. Diese Einheitstheorien stellten dem Namen nach eine einzige Steuer dar, enthielten jedoch eine größere oder geringere Zahl verschiedener Steuerformen.

Der Heidelberger Professor Friedrich Seeger hat die Bedeutung eines aus einer Anzahl direkter Steuern zusammengestellten Systems der Besteuerung zum ersten Mal erkannt und wies auf die Funktionen einer Ausgleichsteuer hin, die den ungleichen Belastungen entgegenwirken sollte. Auf der Grundlage der Lehre von Adam Smith entwickelte Johann Friedrich Eusebius Lotz ein System der direkten Besteuerung, mit dem er das gesamte Reineinkommen des Steuerpflichtigen im Wesentlichen erfassen und die Belastung gleichmäßig und allgemein durchführen wollte. Ludwig Heinrich von Jakob stellte das erste einheitlich geschlossene Besteuerungssystem dar, das den Versuch einer folgerichtigen Verbindung von direkten und indirekten Steuern unter einem einheitlichen Gesichtspunkt bedeutete. Dieses System blieb auf Jahrzehnte hinaus die maßgebende Grundlage der Theorie des

539 Davidsohn, Lars: Verfassungsrechtliche Würdigung, S. 12 f. 
Steuersystems. Den Abschluss dieser Entwicklung bildeten die Überlegungen von Karl Heinrich Rau, der hervorhob, dass alle einzelnen Steuern als Teile eines Ganzen gesehen werden und die Steuerbelastung der verschiedenen Einkommensarten in ein richtiges Verhältnis zueinander gesetzt werden müssten.

Die Autoren, die das Ideal des absoluten Steuersystems der Ertragsteuern als erstrebenswertes Ziel darstellten, verkannten jedoch nicht die Kluft, die sie von der Praxis der Besteuerung trennte und sie hinderte, der Realität gerecht zu werden. In den ersten theoretischen Ansätzen einer subjektiven allgemeinen Einkommensteuer trat das Bestreben in den Vordergrund, die persönlichen Verhältnisse der Steuerpflichtigen zu berücksichtigen. Deshalb schien die allgemeine Einkommensteuer zunächst subjektiv zu sein. In Wirklichkeit war die Theorie der Einkommensteuer bis in die Mitte des 19. Jahrhunderts hinein nichts anderes als eine rechnerische Zusammenfassung der verschiedenen Ertragsteuern.

Die Grund-, Gebäude- und die Gewerbekataster erwiesen sich als schwerfällig und für eine gleichmäßige Besteuerung ungeeignet. Da diese nur zögerlich gepflegt wurden und damit zunehmend von den wirklichen Verhältnissen abwichen, entsprach die Steuerverteilung nach den Katastern nicht mehr der steuerlichen Leistungsfähigkeit der Objekte. Damit wurde die objektive Ertragsteuer zu einer ungerechten Steuer.

Die Steuerreformen zwischen 1818 und 1822 in Preußen erwiesen sich als fiskalisch sehr ergiebig, doch bewirkte insbesondere die Mahl- und Schlachtsteuer eine große steuerliche Ungleichheit. Einerseits erfolgte eine unterschiedliche Besteuerung der Menschen, je nachdem, ob sie auf dem Land oder in der Stadt wohnten, andererseits erwies sich diese Steuer als unsozial, da die ärmere Bevölkerung nur die Möglichkeit hatte, auf Massengrundnahrungsmittel auszuweichen oder auf Konsum zu verzichten. Fernerhin erfolgte teilweise eine doppelte Besteuerung aus Klassen- und Mahl- und Schlachtsteuer, die abhängig vom Wohnsitz in einer Stadt war.

\section{Zum Prinzip der Besteuerung nach der Leistungsfähigkeit}

Die objektiven Ertragsteuern, die die Erträge aus den drei Quellen Grund und Boden, Gebäude und Gewerbebetriebe erfassten, waren typisch für das 19. Jahrhundert in Europa. Die Leistungsfähigkeit konnte nur anhand der äußerlich erkennbaren objektiven Merkmale für den Ertrag des Besitzes eines Bürgers geschätzt werden. Die Steuer haftete auf dem Objekt ohne Berücksichtigung der persönlichen Verhältnisse des steuerpflichtigen Eigentümers oder Nutznießers. Der Gesetzgeber glaubte, auf diese Weise das Problem der Steuergerechtigkeit objektiv und ohne Ansehen der Person gelöst zu haben, bei vollem Schutz der Privatheit des Bürgers. Da der Eigentümer eines steuerbaren Objektes nur nach dem durchschnittlich-möglichen 
Ertrag besteuert wurde, wurde der Fleißige begünstigt, während der Bürger, der aus der Bewirtschaftung seines Steuerobjektes unterdurchschnittliche Erträge erzielte, benachteiligt wurde, denn er wurde so hoch besteuert, als ob er einen durchschnittlich möglichen Ertrag erzielt hätte.

\section{Zum Redistributionspostulat oder zum Grundsatz der steuerlichen Umverteilung von Einkommen und Vermögen}

Bis zur Mitte des 19. Jahrhunderts wurde die Ungerechtigkeit und die Unmöglichkeit einer allgemeinen Einkommensteuer immer wieder betont. In England und Amerika verbreitete sich eine eindrucksvolle Lehre unter der Bezeichnung „leavethem-as-you-find-them-rule of taxation", die als klare Absage an die Progression verstanden wurde.

Dieses Buch wird unter der Creative Commons Namensnennung 4.0 International Lizenz (http://creativecommons.org/licenses/by/4.0/deed.de) veröffentlicht, welche die Nutzung, Vervielfältigung, Bearbeitung, Verbreitung und Wiedergabe in jeglichem Medium und Format erlaubt, sofern Sie den/die ursprünglichen Autor(en) und die Quelle ordnungsgemäß nennen, einen Link zur Creative Commons Lizenz beifügen und angeben, ob Änderungen vorgenommen wurden.

Die in diesem Buch enthaltenen Bilder und sonstiges Drittmaterial unterliegen ebenfalls der genannten Creative Commons Lizenz, sofern sich aus der Abbildungslegende nichts anderes ergibt. Sofern das betreffende Material nicht unter der genannten Creative Commons Lizenz steht und die betreffende Handlung nicht nach gesetzlichen Vorschriften erlaubt ist, ist für die oben aufgeführten Weiterverwendungen des Materials die Einwilligung des jeweiligen Rechteinhabers einzuholen.

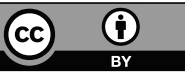

